\title{
Gradhiva
}

GRADHIV

Revue d'anthropologie et d'histoire des arts

16 | 2012

Chines, l'État au musée

\section{L'injonction à la fête. Enjeux locaux patrimoniaux d'une fête en voie de disparition}

An Obligation to Party. Local Heritage at Stake in a Dying Festival

\section{Stéphane Gros}

\section{(2) OpenEdition}

\section{Journals}

Édition électronique

URL : http://journals.openedition.org/gradhiva/2443

DOI : $10.4000 /$ gradhiva.2443

ISSN : 1760-849X

Éditeur

Musée du quai Branly Jacques Chirac

Édition imprimée

Date de publication : 1 décembre 2012

Pagination : 25-43

ISBN : 978-2-35744-048-7

ISSN : 0764-8928

Référence électronique

Stéphane Gros, «L'injonction à la fête. Enjeux locaux patrimoniaux d'une fête en voie de disparition », Gradhiva [En ligne], 16 | 2012, mis en ligne le 01 décembre 2015, consulté le 19 avril 2019. URL : http:// journals.openedition.org/gradhiva/2443; DOI : 10.4000/gradhiva.2443

(c) musée du quai Branly 


\section{L'injonction à la fête}

Enjeux locaux patrimoniaux d'une fête en voie de disparition

\section{par Stéphane Gros}

Comme les autres minorités de la République populaire de Chine, les Drung (Dulong) ont leur propre «festival», officiellement répertorié sous le nom chinois de «Kaquewa» et désormais listé dans l'inventaire national du patrimoine culturel immatériel. Une double officialisation paradoxale: à ce jour ladite fête n'est plus pratiquée par les intéressés.

Cet article explore le contexte particulier de la disparition de cette cérémonie et du rituel qui lui est lié, mais aussi sa brève résurgence suite à un processus de négociation entre les villageois et les représentants locaux du pouvoir, et avec l'ethnologue.

Réponse éphémère à l'injonction officielle à la fête, sa réinstitution engage les acteurs impliqués dans une nécessaire reconstruction de la «culture» drung, qui questionne le rôle de la communauté de référence et son rapport à la tradition d'une part, et pose le problème de la transmission et de la disparition de l'autre. 


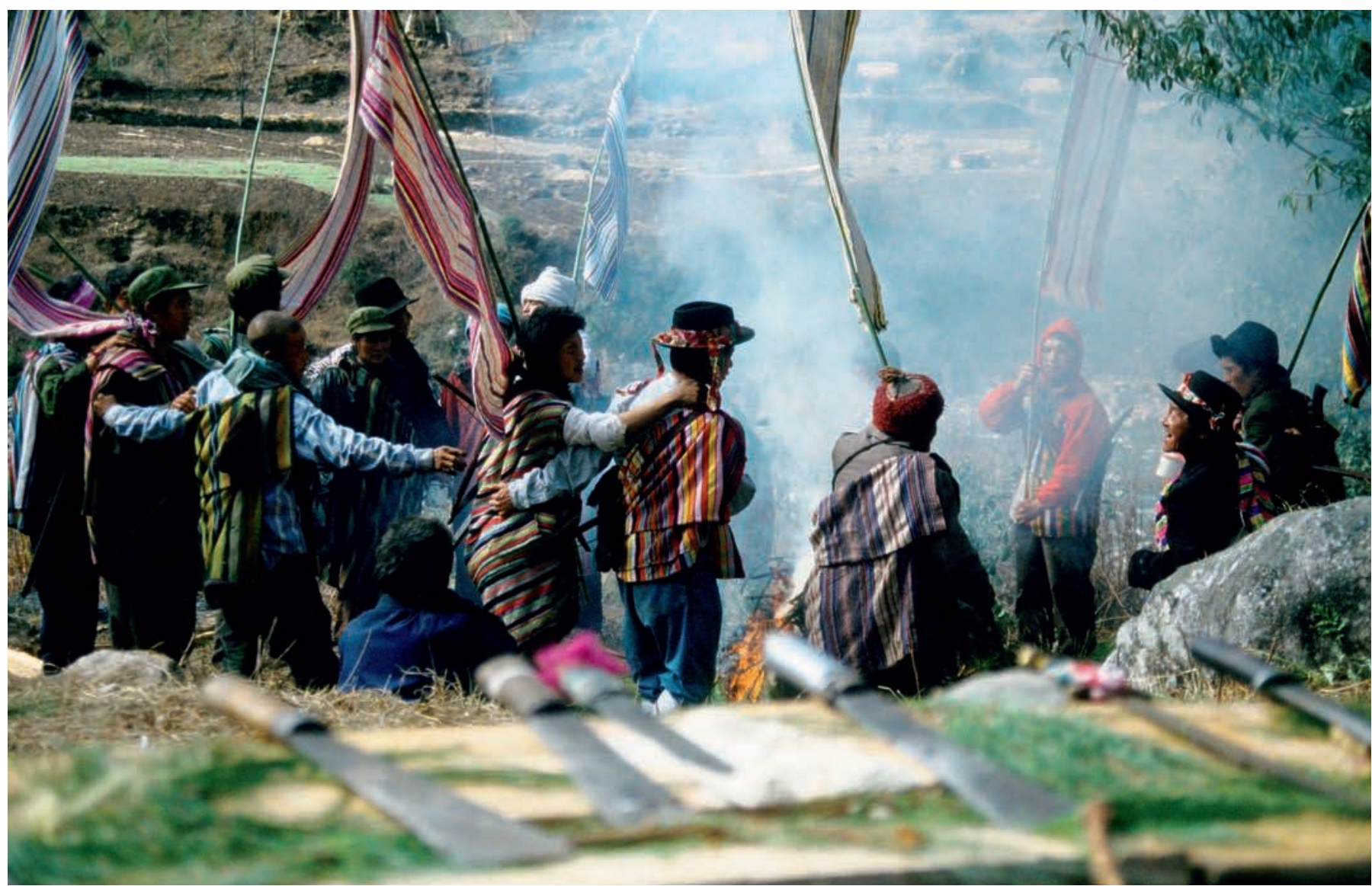

page précédente et ci-dessus

fig. 1

Incantation collective préparatoire à la procession rituelle, 2001.

Photo Stéphane Gros.

\section{Introduction}

En République populaire de Chine (RPC), le «patrimoine culturel immatériel» ( $\mathrm{PCl}$, fei wuzhi wenhua yichan) occupe désormais une place centrale en tant que notion à même d'organiser la préservation des cultures traditionnelles. Signataire de la Convention pour la sauvegarde du patrimoine culturel immatériel de l'Unesco de 2003, la RPC a rapidement engagé un recensement dudit $\mathrm{PCl}$. Cet empressement à compiler des inventaires en vue d'une reconnaissance nationale et internationale est lui-même en continuité avec une plus longue expérience historique, une posture «traditionaliste» (Trébinjac 1990) de sélection des pratiques culturelles devenant emblèmes des cultures populaires. L'instauration de ce rapport à la «culture » - avant même qu'apparaisse la notion de patrimoine - a pris une forme moderne autant que politique et, depuis les années 1950, diverses instances bureaucratiques qui ont vu le jour ont été mobilisées pour l'action culturelle. Début 2006, l'Administration d'État du patrimoine culturel (ancien Bureau d'État des reliques culturelles), relevant du ministère de la Culture, a reçu de nombreuses candidatures transmises par les districts, préfectures et régions en vue de l'établissement d'un inventaire national du patrimoine culturel immatériel. Une première liste, diffusée par une circulaire du Conseil des affaires d’État en janvier 2006, comptait 501 éléments. Elle fut révisée en mai de la même année pour constituer une liste définitive de 518 éléments classés en 10 catégories (Conseil des affaires d'État 2006; Barmé 2006a, 2006b; Pan 2008: 15). Au sein de cette liste, 146 éléments émanent 
des cultures des «nationalités minoritaires» (shaoshu minzu), qui représentent à ce jour environ $8 \%$ de la population totale du pays. Dans la section 10 («Coutumes», minsu) de cet inventaire national figure l'élément $n^{\circ} 471$, «Fête Kaquewa de la nationalité dulong [drung] » (dulongzu kaquewa jie ${ }^{1}$ ).

Cette fête, généralement connue sous le nom de «Kaquewa» (transcription en chinois de son nom drung, kraltshang-wa), fut reconnue et officialisée dès 1991 sur décision du gouvernement du district autonome de Gongshan, où les Drung sont majoritairement présents. Le 10 janvier en est la date officielle. Son classement au sein de la liste du $\mathrm{PCl}$ n'est que la confirmation d'une taxonomie et d'une normalisation culturelle préexistantes (Nas 2002). Comme les autres minorités du pays, les Drung ont ainsi leur propre «fête», mentionnée quasi systématiquement dans les divers documents présentant la culture drung au grand public. Le rituel, devenu fête, est un objet patrimonial par excellence (Brosius et Polit 2011). Grâce à son inclusion dans la liste nationale du patrimoine immatériel, le Kaquewa a acquis un degré supplémentaire de visibilité et, en somme, une valeur ajoutée.

D'emblée surgit le problème de la relation entre une décision politique et la continuité d'une tradition locale. En effet, le Kaquewa est une célébration rituelle du Nouvel An qui fut interdite par le pouvoir en place pendant près de vingt ans. Paradoxalement, il est désormais officialisé alors qu'il n'est plus pratiqué par les intéressés.

Cette officialisation et promotion par inventaire pourrait être sans conséquence, et le Kaquewa ne demeurer qu'un nom sur une liste faisant étalage de la richesse des ressources culturelles des minorités - artificialité d'une fête sans référent sur laquelle je me pencherai. L'inventaire ne fut en somme qu'une question de forme, non un changement de régime culturel. Ce sont donc les précédents de cette «nouvelle» catégorie d'action politique qu'est le $\mathrm{PCl}$ qu'il importe de mettre au jour, en prenant pour point de départ l'histoire locale ${ }^{2}$. Car l'«injonction » officielle à la fête, lancée dès le début des années 1990, résonna jusque dans la vallée reculée des montagnes du nord-ouest de la province du Yunnan où habitent les Drung.

Le rituel (kraltshang-wa) fut interdit pendant la Révolution culturelle, à partir de 1966, en tant que pratique «superstitieuse». Suite à la libéralisation politique du début des années 1980, l'attitude officielle concernant les pratiques religieuses évolua, et les autorités furent plus conciliantes. Dans maintes régions de Chine, ce fut un vaste mouvement de résurgence des cultes, parfois à l'initiative même des autorités locales. Pour les Drung, ce processus n'eut pas cet aspect spontané et spectaculaire. Ce n'est qu'en 1991 que le gouvernement du district autonome de Gongshan «officialisa » la fête drung, et seuls certains villages du nord de la vallée pratiquèrent à nouveau le rituel en question. Le mouvement ne fut que de courte durée. De leur propre chef, les habitants décidèrent de ne pas perpétuer cet usage, pour des raisons essentiellement économiques semble-t-il. Quelques années plus tard, il n'y avait ainsi plus de fête annuelle qui puisse servir de symbole de la tradition drung. La «fête drung » fut pourtant officialisée précisément afin qu'un tel marqueur identitaire perdure.
1. Ce numéro d'inventaire est celui attribué sur la seconde liste. La première référençait cette fête sous le $n^{\circ} 462$.

2. Pour une récente discussion de la notion de patrimoine culturel immatériel, voir plus particulièrement Bortolotto (2011). 
Dans un village au nord de la vallée des Drung, il y eut un bref sursaut qui mena à la réinstitution provisoire de cette fête au début des années 2000 . II n'était pas encore question de $\mathrm{PCl}$, mais cette «nouvelle» notion n'était pas nécessaire dans un régime culturel interventionniste où la portée politique de l'action patrimoniale était affaire courante. Cet article détaille le processus de négociation qui eut alors lieu au niveau villageois. L'État et son intervention dans le domaine culturel apparaîtront à travers la figure d'un de ses agents, un secrétaire du Parti, mais aussi dans l'enchevêtrement des discours et des enjeux liés à la valorisation d'un tel événement. Car après cette éphémère réponse à l'injonction, la fête a bien disparu. Si le rituel qui est la raison d'être du Kaquewa n'est plus effectué, quel contenu attribuer à cette cérémonie désormais inventoriée? Les questions de transmission et de disparition mises en jeu par la notion même de patrimoine immatériel doivent être discutées, et le possible devenir de la fête drung interrogé.

Le cas du Kaquewa illustre les enjeux concrets de transmission et de reconnaissance qui se sont posés pour les acteurs impliqués dans la reprise provisoire de ce qui fut, autrefois, une tradition vivante. II touche à ce moment crucial d'objectivation de la culture, de transformation d'une pratique en un bien-valeur. De la fête, désormais débarrassée du rituel, que reste-t-il?

\section{La fête aura-t-elle lieu?}

Au cours de mes enquêtes de terrain entreprises à la fin des années 1990 dans le nord de la vallée des Drung, à Dizhengdang, j'eus de nombreuses occasions de discuter du kraltshang-wa, et notamment de sa disparition, avec les villageois. L'un en particulier m'avait assuré plusieurs fois qu'il désirait le réinstituer: c'était Li Zisheng, le secrétaire de cellule du Parti (zhibu shuji) du village. Au printemps 2000, je me souviens l'avoir trouvé en discussion avec son frère chamane et chef de la commune (shezhang): tous deux m'assurèrent que l'année suivante ils feraient le kraltshang-wa.

Sans doute ma seule présence constituait-elle une demande, une incitation pressante, car tous au village connaissaient mon intérêt pour les différents aspects de leur vie quotidienne. N'était-ce pas là une manière de me dire que beaucoup restait à voir et à comprendre, d'affirmer un désir de reconnaissance tout comme la vitalité de certaines pratiques? L'implication du secrétaire du Parti semblait décisive, et Li Zisheng m'engagea à venir I'hiver suivant pour assister à la cérémonie.

J'organisai un nouveau séjour et, cette fois, m'équipai d'une caméra vidéo dans le but de filmer la fête du Nouvel An - l'électricité venait d'arriver au village grâce à la construction d'une petite station hydraulique. Le soir même de mon arrivée, le 3 décembre 2000, je trouvai le secrétaire du Parti dans une pièce du bâtiment public, assis devant une petite télévision en noir et blanc. Son attitude était fuyante. Quelques jours passèrent et je questionnai mes amis sur la situation. Finalement, le 10 décembre, fut organisée une réunion entre le chef du village (cunzhang), le secrétaire du Parti et le chef de la commune (tous des villageois drung), pour discuter de l'organisation de la fête. II fut alors convenu de tenir une petite assemblée avec tous les villageois le soir suivant. 

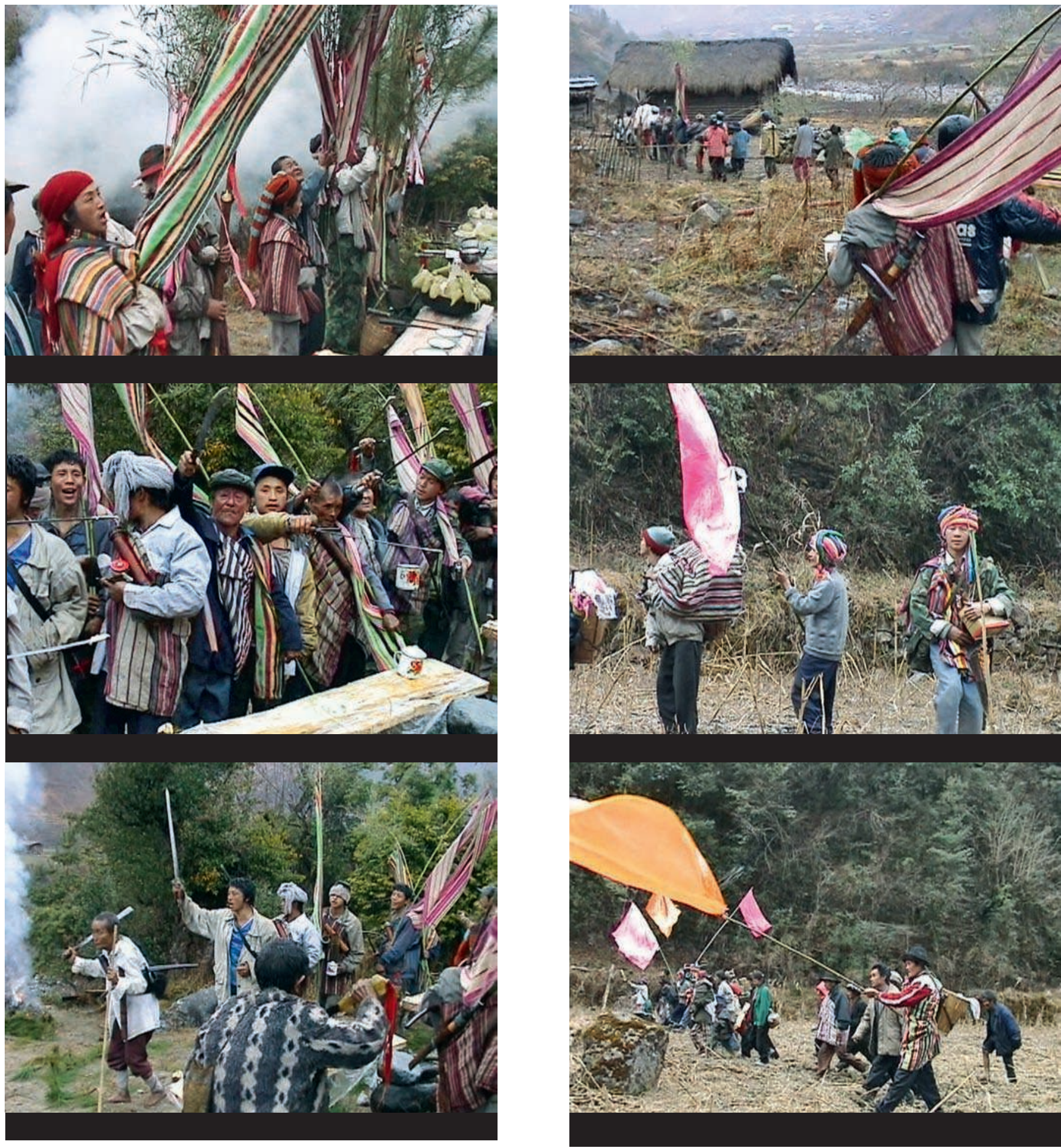

fig. 2

Offrande de drapeaux

rituels, procession (2001)

et défilé avec «drapeaux

rouges » (2003) durant 
3. La langue drung fut utilisée lors de cette réunion, aussi les occurrences significatives des termes chinois sont signalées entre parenthèses.

4. Il est fait ici allusion au calendrier officiel des fêtes des nationalités minoritaires. Le Nouvel An drung, sous le nom chinois de "Kaquewa", a ainsi été arbitrairement fixé au 10 janvier de chaque année, comme je l'ai précisé plus haut.

5. Sur le problème de l'implication de l'ethnologue dans l'action patrimoniale, voir par exemple Barbe (2007) et Tornatore (2007).

6. J'ai systématiquement organisé des visionnages de ce que je filmais.

Les questions éthiques et méthodologiques soulevées par cette situation ont été traitées dans un autre article, voir Gros (2010)
Le lendemain, dans la maison du chef de la commune, autour du foyer, peu d'habitants étaient présents. Je fus invité à parler et, m'exprimant en chinois pour plus d'aisance, expliquai que je revenais cette année parce qu'il m'avait été dit que le kraltshang-wa aurait lieu; je ne venais pas demander aux villageois d'organiser la fête, mais croyais qu'eux-mêmes le souhaitaient. Quand les responsables prirent la parole et signifièrent leur volonté de célébrer à nouveau cette fête, personne ne donna son avis. II fut décidé de reporter la réunion au lendemain soir, en espérant plus de monde.

Le 12 décembre, toujours chez le chef de la commune, presque toutes les maisonnées étaient représentées; mais peu d'hommes s'étaient déplacés, laissant aux femmes la charge d'assister à cette réunion. Tout le monde était assis autour du feu dont la lumière vacillante éclairait les visages. Les responsables prirent la parole, l'un après l'autre ${ }^{3}$. Le secrétaire du Parti : « [...] II vient étudier la nationalité (minzu) drung, la culture (wenhua), les coutumes (fengsu), [...] eh bien, cette année il revient pour savoir si on veut ou non faire le kraltshang. Voilà pourquoi il vient. [...] Ce qu'il veut savoir, c'est s'il y a des gens qui veulent le faire; si tout le monde est d'accord et veut le faire, alors il filmera, et plus tard les enfants pourront regarder. Voilà ce qu'il vient faire. " Ainsi le secrétaire du Parti reformula et compléta à sa manière ce que j'avais dit le soir précédent. Il continua: «Nous, les Drung, comment les vieux faisaient, on ne sait plus. Alors si vous voulez le faire, il recueillera tout, il filmera, et après [...] on pourra regarder comment les vieux faisaient. »

Après un moment de silence, le chef du village prit la parole: «ll vient pour une demi-année voir ce que l'on fait [nos activités], il recueille [les faits] et après, en plein d'endroits des gens pourront savoir. Cela a une grande utilité (zuoyong). [...] La chose la plus importante, c'est le kraltshang. Jadis, dans la vallée drung, des fêtes (jieri) d'une sorte ou d'une autre, il n'y en avait pas. C'est la seule. Maintenant, il a déjà été décidé que le 10 janvier, c'est la fête (jie) kraltshang ${ }^{4}$. [...] »

Comme je le pressentais, ma présence en tant qu'«ethnologue» faisait désormais de moi le promoteur de la fête, sorte de culture broker (Geertz 1960), présenté comme tel par le secrétaire. II ne s'agissait plus de réaliser un souhait émanant de certains des villageois, ou bien même du seul secrétaire du Parti, mais de répondre à une demande extérieure: à l'injonction étatique s'ajoutait le désir de l'ethnologue ${ }^{5}$. La situation s'était renversée. J'étais alors partie prenante dans un processus plus large de promotion d'une «fête» représentative de la «culture» drung. Mes écrits à venir et, mieux encore, la vidéo que j'allais réaliser seraient un atout non seulement pour la diffusion et la valorisation du kraltshang-wa, mais aussi pour assurer la mémoire et la transmission de ce savoir-faire pour les générations futures, les plus jeunes ne sachant pas accomplir ce rituel complexe ${ }^{6}$.

L'usage des termes chinois au cours de cette réunion qui se déroula en langue drung est lui-même significatif. Le travail de l'ethnologue tout comme l'importance de cette «fête » furent évoqués à travers un vocabulaire spécifique révélant la manière dont le discours officiel relatif aux cultures des minorités peut être localement mobilisé. La «fête drung» avait déjà 
été officialisée, et il y avait un enjeu de transmission de la «culture» et des "coutumes» associées au statut officiel de «nationalité». En somme, tous les éléments mis en jeu par la politique culturelle (et patrimoniale) étaient en place: l'importance de l'objet et la légitimité de son choix, la reconnaissance de sa valeur présupposant le droit statutaire à la revendiquer. Un enjeu supplémentaire se profilait, car la promotion du patrimoine culturel va souvent de pair avec le tourisme. Le chef du village continua: "Chaque endroit doit avoir sa particularité (tese); voilà ce qu'il faut faire. Les gens qui voyagent, ils vont là où c'est amusant, où il y a une particularité. [...] »

Après les interventions des dirigeants locaux, les villageois présents restèrent longtemps silencieux. Quand ils commencèrent à prendre la parole, le manque de moyens et de temps pour se préparer ou, pour certains, leur récente conversion au christianisme furent autant de motivations pour ne pas s'engager. Tout laissait présager que la fête n'aurait pas lieu. On faisait de moi l'initiateur de ce projet et je me trouvais directement impliqué. L'idéal de «l'atopie de l'ethnographe ${ }^{\mathbf{7}}$ » était, au moins provisoirement, anéanti. Certains m'exhortaient à intervenir, ce que je refusais de faire. Les choses en étaient restées à la très faible probabilité que le kraltshangwa ait lieu, et le secrétaire du Parti avait en somme été mis en minorité.

II fallut attendre le dénouement plusieurs semaines. Un groupe de villageois s'était désormais déclaré prêt à organiser le rituel. Ceux-ci me présentèrent leur décision comme une réponse à mon désir d'ethnographe: l'amitié les motivait, et ils pourraient ainsi témoigner d'une pratique traditionnelle non encore étudiée par un ethnologue. À leur demande, j'allai trouver les responsables locaux, secrétaire du Parti, chef du village et chefs de commune, pour organiser une nouvelle réunion.

Celle-ci eut lieu le 13 janvier 2001 au soir. II fallait que, de vive voix, les responsables locaux donnent leur accord car ce choix émanant de quelques villageois avait provoqué des tensions révélant que l'enjeu n'était pas seulement la décision politique de ranimer une pratique tombée en désuétude. Des rivalités individuelles, la remise en cause de l'autorité de certains - et notamment du secrétaire Li Zisheng -, les difficultés concernant l'économie familiale, le doute relatif à l'efficacité du rituel, mais aussi la crainte de ne plus savoir, celle d'offenser les esprits, de s'attirer leur sanction... tout un ensemble complexe de déterminants, un contexte conditionnant les possibilités d'action et les intérêts de chacun avait surgi.

Cette dernière réunion permit de clarifier les positions, en particulier par rapport à la légitimité d'une telle démarche. Pourquoi la fête n'avaitelle pas été réinstituée jusqu'alors? Li Zisheng rappela que les habitants, quoi qu'il dise afin de les mobiliser, n'écoutaient pas. II précisa néanmoins: "S'il y a quatre ou cinq maisons ça suffit... il faut un esprit d'ouverture (sixiang kaifang) [...]" Son frère, le chef de la commune, l'interpella fermement: "Ce sont les cadres dirigeants (lingdao ganbu) qui décident, non? [...] Qu'est-ce qu'il y a à craindre? Voilà ce que les chefs doivent dire! [...]»

Le porte-parole du groupe ayant pris la décision d'organiser le rituel du Nouvel An - l'aîné de ce groupe lignager qui, de manière signi-
7. J'emprunte ici la formule par laquelle Naepels (1998) caractérise l'idéal de toute enquête (sociologique ou ethnographique). 
ficative, avait lui-même été secrétaire du Parti autrefois - déclara alors, en employant essentiellement des formules en chinois: «Les autorités de la sous-préfecture, de la préfecture, ont dit: "Faites-le, il faut faire vos propres coutumes de nationalité (minzu xiguan)"... nos propres particularités de nationalité (minzu tedian), il faut les faire. [...] II faut nous-mêmes rétablir (huifu) nos particularités de nationalité. [...] Le problème clé, ce sont les dirigeants, vous deux, vous n'êtes pas assez passés à l'action (tuixing). Le peuple doit être mobilisé (renmin yao fadong qilai). " Le chef de la commune: «Oui, les dirigeants doivent mobiliser les habitants! " Le secrétaire du Parti: «Mais on a essayé et on n'arrive pas à les mobiliser! J'ai dit ce qu'il fallait lors des réunions!»

Le villageois rappela que c'était l'assemblée populaire (renda) qui avait déclaré que la fête devait être le 10 janvier pour tout le canton - l'ensemble de la vallée des Drung -, et que les autorités prévoyaient même de donner de l'argent... II poursuivit: «Voilà ce que je pense... Vous, dirigeants, est-ce que vous avez bien parlé? Est-ce que vous avez dit ce qu'il fallait? [...] Que ceux qui veulent le faire le fassent, que ceux qui ne le font pas ne s'y opposent (fandui) pas! " Le chef de la commune: «Voilà qui est bien dit. " Le secrétaire du Parti: «C'est un usage de notre nationalité (minzu xiguan), pourquoi on s'y opposerait? »

La réunion se termina peu de temps après. Je n'étais pas sûr qu'elle ait vraiment abouti et fus surpris de voir le secrétaire du Parti manifestement réjoui. L'essentiel avait donc été dit. II apporta une bouteille d'alcool pour fêter cela.

Il était évident que l'éventualité d'accomplir le rituel du Nouvel An devenait une source potentielle de conflit. II fallait certes ménager le secrétaire du Parti, initiateur de l'idée, qui pensait pouvoir décider d'une action collective et «mobiliser » tout le monde, comme il le faisait à l'occasion des travaux d'intérêt public. Or il n'y parvenait pas. Tout sentiment de rivalité ou de jalousie devait être dissipé. En arrière-plan de ces discussions, le problème restait celui de la volonté toute relative de la plupart des villageois de faire le kraltshang-wa. Dans leur ensemble, les villageois étaient très réticents à la reprise du rituel du Nouvel An, car faire de nouveau appel aux esprits comportait un danger. Les plus jeunes en particulier craignaient de ne pas suffisamment maîtriser les longues incantations. On me donna l'exemple d'un hameau du centre de la vallée où pratiquement tous les habitants avaient trouvé la mort suite à un kraltshang-wa probablement mal exécuté.

Les villageois mentionnaient quasi systématiquement l'investissement économique important qu'impliquait la préparation du rituel, auquel s'ajoutait l'épreuve physique de la fatigue. L'économie familiale était d'autant plus un sujet de préoccupation que, depuis la fin des années 1990, des plans de développement gouvernementaux s'étaient succédé et les discours sur la rentabilité agricole, la bonne gestion et l'épargne avaient exercé une influence indéniable. Outre ces aspects économiques, l'intérêt de l'action collective de même que l'efficacité du rituel étaient questionnés. II n'y avait plus de volonté collective, comme ce fut le cas au début des années 1990 lorsque les habitants 


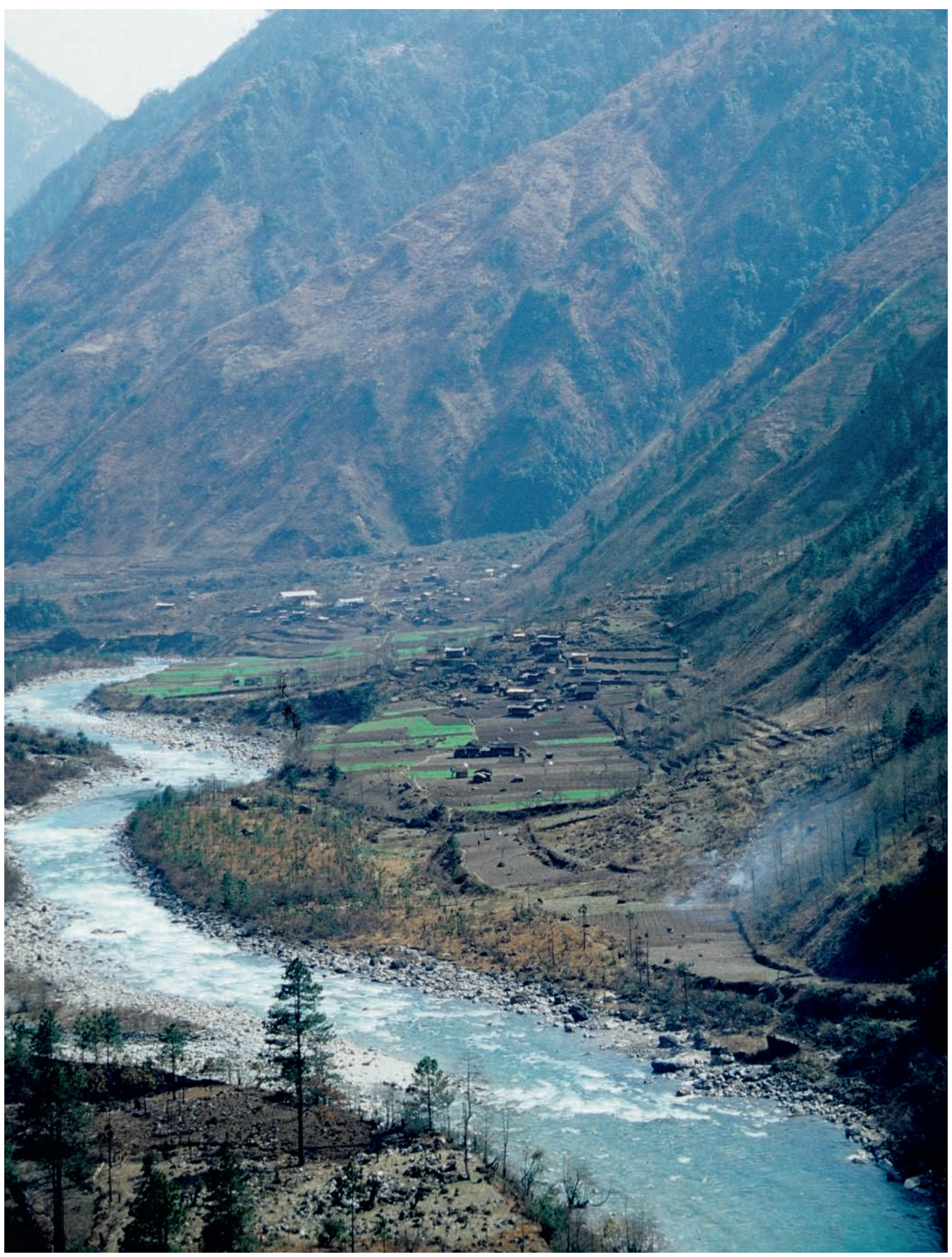




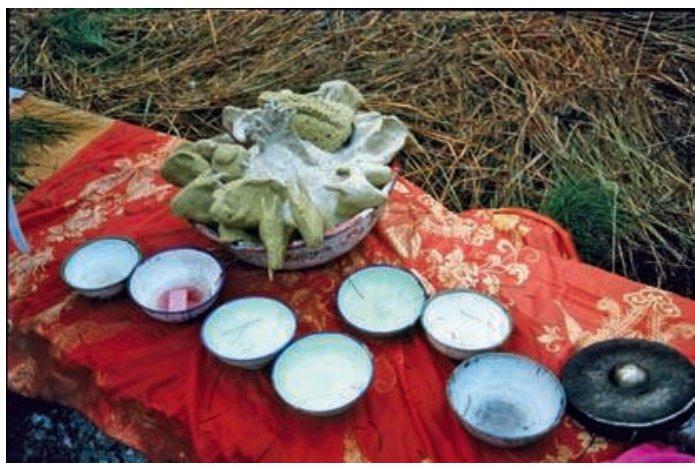

fig. 4

Figurines rituelles et bols de divination sur l'autel, 2003. Photo Stéphane Gros. de Dizhengdang refirent le kraltshang-wa durant quelques années, après l'avoir abandonné pendant près de trois décennies. La vie religieuse reposait désormais uniquement sur la gestion des aléas de l'infortune individuelle.

Voilà le kraltshang-wa replacé dans son contexte particulier: non pas la perpétuation d'une tradition ancestrale mais une négociation entre les villageois eux-mêmes et avec les représentants locaux du pouvoir (le secrétaire du Parti, le chef du village, les chefs de commune...), négociation dont l'un des enjeux fut la reconstruction de ce qui doit être constitutif de la «culture» drung. De toute évidence, le kraltshang-wa n'était plus une pratique qu'animait un devoir collectif de perpétuation. Au-delà du référent de la «nationalité », cadre officiel de l'expression identitaire, la communauté de référence ne présentait pas plus de sentiment d'unité et d'identité à travers un souhait collectif, idéal de la nouvelle politique patrimoniale. Ce furent au contraire les dissensions internes, propres à l'histoire locale et aux relations liant les divers acteurs, qui émergèrent. De multiples lignes d'interprétation pourraient certes être suivies pour démêler les enjeux qui se cristallisèrent à cette occasion ${ }^{8}$. II m'importe ici uniquement de traiter de la question du devenir du rituel suite à sa réinstauration.

\section{Retour aux traditions}

Au village de Dizhengdang, seule une minorité de villageois décida de fêter à nouveau le Nouvel An pendant trois années consécutives (2001-2003), conformément à l'obligation rituelle de le célébrer pendant un nombre d'années qui soit un multiple de trois. Le secrétaire Li Zisheng vint y assister, mais il ne fut que spectateur. Initiateur, il s'était néanmoins dégagé de toute responsabilité dans son organisation, désormais prise en main par les villageois (essentiellement un groupe lignager et leurs proches voisins et amis) qui s'étaient portés volontaires.

La première année fut d'emblée placée sous le sceau du traditionnel: il s'agissait de faire "comme les Anciens" et de renouer sous cette forme avec la cérémonie du kraltshang-wa qui allait durer trois jours. L'organisation du Nouvel An, cet hiver 2001, fut ainsi encadrée par les plus âgés. Lors des diverses phases rituelles, ce furent eux qui récitèrent les incantations. Des drapeaux rituels furent confectionnés comme il se doit au moyen de longues étoffes tissées par les femmes, «monnaie» d'échange avec les esprits ${ }^{9}$. Chaque maisonnée participante prépara en quantité de la bière de céréales fermentées et confectionna les figurines en pâte de farine de sarrasin indispensables à la bonne tenue du rituel (fig. 4). Une aire rituelle fut aménagée, où l'essentiel des séquences d'offrandes prit place. L'ensemble se déroula alors selon un agencement orthodoxe: séquences divinatoires, incantations, danses, pour enfin recevoir la «bonne fortune» des esprits et s'empresser d'aller la déposer dans les maisons.

Les réjouissances, accompagnées d'une importante consommation de bière fermentée, furent ponctuées de danses et de chants, mais aussi de jeux essentiellement masculins: courses de vitesse, concours de saut et, surtout, de tir à l'arbalète. Enfin, un repas communautaire eut lieu sur l'aire rituelle. Chacun eut sa part, gage de longévité, y compris les invités, tel le secrétaire Li Zisheng ou moi-même, l'ethnologue de service. 
Ce fut alors le dernier temps fort de la cérémonie: les hommes s'équipèrent de leurs arbalètes et carquois, et prirent en main leurs épées rituelles. Se réunissant devant l'autel, ils invoquèrent un esprit protecteur, et la file des hommes en armes portant les drapeaux rituels se transforma en une procession qui traversa le village. Tout le monde se devait d'intégrer celle-ci pour raccompagner les puissants esprits (fig. 2) qui avaient été conviés.

Le lendemain, dernier jour de la fête, du bois de chauffage, du maïs et du soja furent mis en commun puis partagés, soulignant l'importance de la commensalité, déjà évidente lors du repas commun à l'aire rituelle. Et pour clore ces trois jours de célébration, des danses se déroulèrent successivement dans chacune des maisonnées participantes.

Ce souci de bien faire était motivé par l'importance accordée à l'apprentissage par les plus jeunes adultes, et par la volonté de présenter à l'ethnologue et à la caméra une version «authentique» du rituel. Moment d'objectivation et de performance, sorte de retour «métadiscursif» sur la tradition (Briggs 1996) qui poussa certains à revêtir pour l'occasion une tenue proprement drung, «à l'ancienne». Si bien que lorsque quelques-uns décidèrent d'allumer la télévision et de chanter en karaoké ${ }^{\mathbf{1 0}}$, ce fut l'objet d'une sérieuse dispute entre les partisans du chant traditionnel et ceux de la musique moderne chinoise. Parmi ces derniers, l'un fut particulièrement vexé: «Et pourquoi ne pourrait-on pas s’amuser comme ça? » répétait-il.

\section{Place aux jeunes}

À mon arrivée à Dizhengdang en décembre 2002, alors que je devais assister à la troisième et dernière année du rituel de Nouvel An, il me fut tôt déclaré: «Maintenant, c'est l'affaire des jeunes. » Désormais la nouvelle génération devait prendre les choses en main.

Cette année-là, le kraltshang-wa fut fêté à la date du Nouvel An chinois (le $1^{\text {er }}$ février), comme cela avait été le cas l'année précédente. Le même groupe de maisonnées participa au rituel, et l'ensemble de la cérémonie se déroula selon la trame des séquences rituelles et festives. Si la phase proprement rituelle resta inchangée dans sa succession de séquences formalisées, un ensemble de pratiques témoignèrent d'un processus de transformation. Cibles de l'apprentissage les deux années précédentes, les jeunes allaient maintenant innover, selon une grammaire des possibles, et fabriquer de la fête.

Tout d'abord, un signe emblématique de l'esprit qui anima la réalisation du rituel fut la confection d'un nouvel élément: les participants décorèrent un arbre qui se trouvait derrière l'autel et qu'ils désignèrent du nom d'«arbre rituel commun». Celui-ci symbolisait l'unité des participants qui, depuis 2001, faisaient communauté autour du rituel; il constituait également, au dire des villageois, un élément censé réjouir les esprits pour cette dernière année du kraltshang-wa.

L'expression de la solidarité entre les participants marqua, en effet, les festivités. Le dernier jour de célébration, au partage conventionnel de maîs et de soja s'ajouta une collecte en nature organisée pour la prépa-
8. C'est là l'objet d'un ouvrage en préparation.

9. Pour une description et une discussion du rituel du Nouvel An, voir Gros 2012b

10. Il y avait de l'électricité au village depuis un an, et déjà quelques foyers s'étaient équipés d'une télévision et du nécessaire pour regarder des vidéos et chanter en karaoké. 
ration d'un grand repas en commun. Cette initiative avait été prise l'année précédente par les jeunes et fut dès lors vivement approuvée par tous les participants.

Ce fut également à l'initiative des jeunes que prit place, le lendemain, un grand "pique-nique» à l'extérieur du village. Quatre personnes se munirent de grands drapeaux confectionnés pour l'occasion, qu'ils appelèrent du terme chinois hongqi («drapeaux rouges», tels les drapeaux révolutionnaires). Puis, comme sur le modèle de la procession rituelle qui clôt la cérémonie du Nouvel An et raccompagne les esprits, une longue file indienne se mit en branle, les drapeaux gonflés par le vent, un magnétophone portable hurlant de la musique populaire chinoise (fig. 2).

Au moment d'arriver à l'aire du pique-nique, les quatre «drapeaux rouges» furent fichés dans le sol, délimitant un espace carré, et un arbre rituel, censé contribuer à recueillir la fertilité venant des esprits fut dressé. Une sorte de reproduction condensée de l'aire rituelle fut ainsi élaborée par l'utilisation de quelques éléments symboliquement chargés, tels les drapeaux et l'arbre rituel. Les drapeaux rouges, qui pouvaient se comprendre comme une parodie d'un symbole du pouvoir, eurent donc une finalité inattendue. Comme si cette procession-là se faisait non pas sous la protection des esprits, mais sous celle de l'État.

Ce «pique-nique »futnéanmoins dèsledépartplacésouslesignedujeu. La journée prit un caractère quasi carnavalesque car certains jeunes adultes, hommes comme femmes, avaient décidé de se déguiser. Quelques-uns s'étaient juste vêtus de manière insolite, portant lunettes noires et casquette de l'armée (fig.5), mais des hommes avaient poussé l'originalité jusqu'au travestissement vestimentaire, portant une robe ou une jupe (fig. 6). Peutêtre faut-il rapprocher ce travestissement du «jeu» comme forme rituelle; il prendrait alors sens avec les autres divertissements de cette journée qui se passa à danser sur de la musique disco, à manger et boire, à faire des courses de vitesse entre hommes puis entre femmes. Ces éléments ludiques sont généralement déjà présents lors de la cérémonie sur l'aire rituelle. Pendant le kraltshang-wa, il faut boire, danser et jouer, autant d'actions nécessaires au bon déroulement de l'ensemble.

Après la première année de reprise du rituel, caractérisée par une recherche du traditionnel et de l'authentique, l'effort de transmission avait produit de la «fête». La journée de pique-nique recyclait des éléments propres au rituel tout en intégrant d'autres formes. Bien que sous une apparence anodine et ludique, cette journée avait elle aussi son efficacité. Elle contribua à assurer la cohésion de l'ensemble des participants et leur commune prospérité pour l'année à venir, tout en s'inscrivant dans une dynamique sociale, une nouvelle façon de célébrer le Nouvel An et de faire la «fête», de penser sa place dans le monde d'aujourd'hui.

Le cycle des trois années du kraltshang-wa prit ainsi fin en février 2003. Pour les villageois qui l'avaient provisoirement réinstaurée, cette cérémonie ne s'était pas cantonnée à la répétition d'un «noyau » rituel, mais s'était construite de manière dynamique jusqu'à produire une forme originale. 


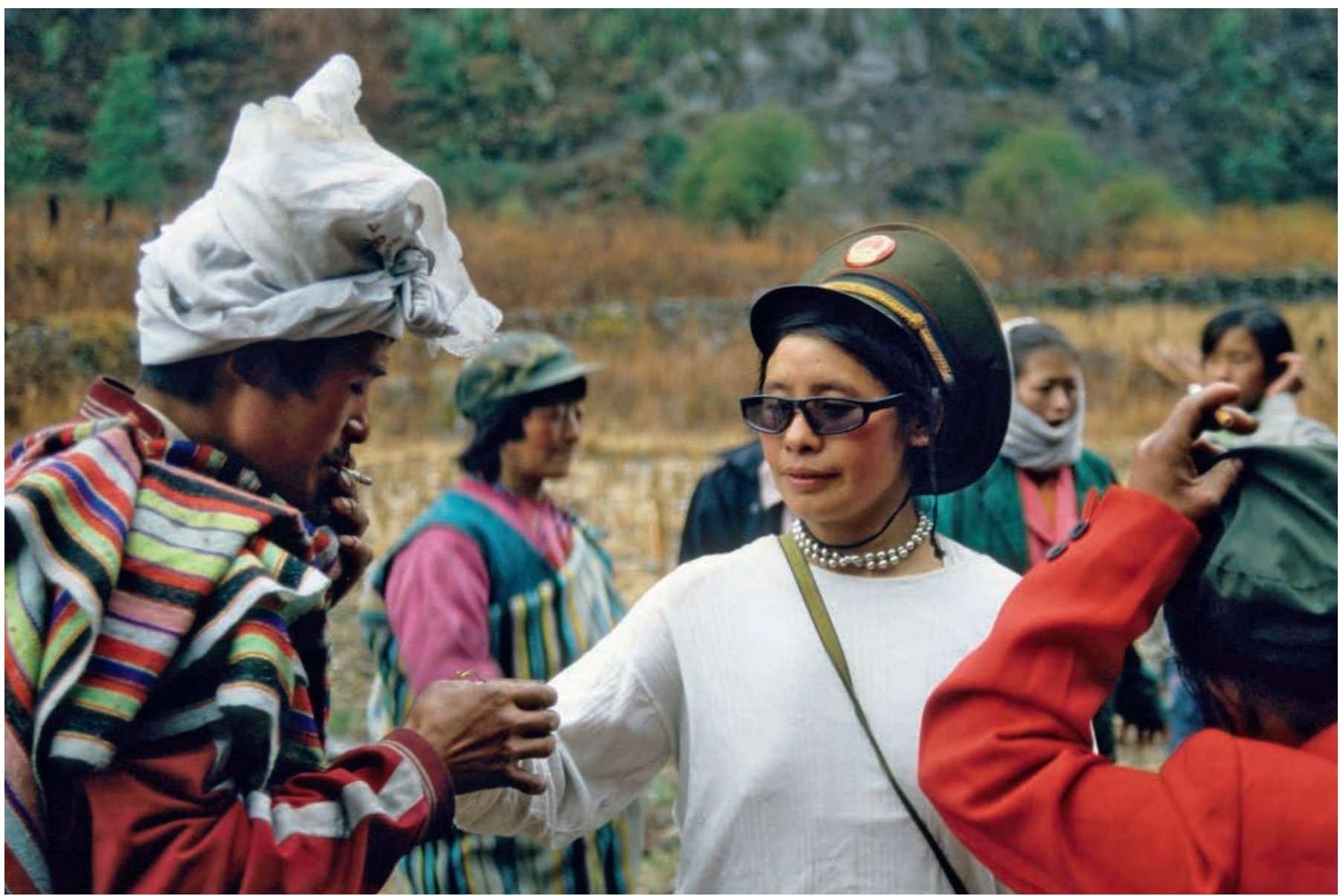

À l'appropriation du rite par la nouvelle génération s'ajouta l'hypertrophie du jeu, de la procession et du travestissement, comme l'emphase sur le partage entre les participants. Dans l'évolution du kraltshang-wa, expression de ce que la cérémonie du Nouvel An devient quand la jeune génération la prend en main, se cristallisent certains éléments d'une «culture de synthèse» dans laquelle se fondent les savoir-faire hérités, des usages objectivés et l'affirmation d'une identité.

Désormais, les villageois de Dizhengdang ne refont plus la cérémonie du Nouvel An qu'ils furent les derniers à organiser dans la vallée des Drung. Le kraltshang-wa s'est donc éteint en 2003. Depuis, son avatar, le «Kaquewa», a néanmoins perduré, inventorié au patrimoine culturel immatériel. Qu'est la fête drung devenue? Cette question de la perpétuation, ou de la fabrication de la fête, demande à revenir sur l'encadrement institutionnel et les outils mis en place pour assurer la continuité dudit bien patrimonial, et en premier lieu au rôle clé dévolu aux personnes désignées comme «transmetteurs» de ce patrimoine culturel pour leur communauté. fig. 5

Les jeunes font la fête, 2003. Photo Stéphane Gros. 
11. Cette nouveauté par rapport à la notion de patrimoine bâti qui prévalait alors, a été très largement soulignée et discutée dans nombre de publications. Le problème de la traduction de cette notion a en Chine comme ailleurs causé débat. Pour une approche générale de la notion de patrimoine en Chine, voir Fresnais (2001) et Zhang (2003).

12. Selon la phraséologie officielle où s'élaborent à la fois un socialisme et une économie de marché "avec des caractéristiques chinoises".

13. Cette loi, votée le 25 février 2011 et entrée en application le $1^{\text {er }}$ juin 2011 , précise tout d'abord que son objectif premier est de "promouvoir l'édification de la civilisation spirituelle du socialisme, le travail de protection et de sauvegarde du patrimoine culturel immatériel » (article 1).

14. À partir de cette liste, le ministère de la Culture a alors sélectionné un total de 32 transmetteurs de rang national pour la province du Yunnan.

\section{Un "transmetteur" pas comme les autres}

La définition donnée par l'Unesco place le patrimoine culturel immatériel au confluent de préoccupations hétérogènes. Le $\mathrm{PCl}$ opère en effet un renversement par rapport à la notion d'objet ${ }^{\mathbf{1 1}}$, et l'article 2 de la convention implique le soutien apporté aux communautés par les États en vue de la sauvegarde de ce patrimoine, tout en insistant par ailleurs sur la transmission entre les générations et le processus afférant de recréation (voir Bortolotto 2011). Le $\mathrm{PCl}$ apparaît certes comme une notion à même de prendre en compte une réalité vivante et mouvante, mais également et avant tout peut-être comme une notion nécessairement politique.

Le paradoxe de l'immatériel, lorsqu'il s'associe à la notion de patrimoine et à la volonté de le sauvegarder, repose sur la nécessité de l'incorporer dans un «bien». Cet être invisible au cœur de la dynamique culturelle doit donc prendre forme pour être saisi par l'action publique patrimoniale. C'est ainsi que le patrimoine immatériel peut s'incarner, au sens propre, dans des personnes. Ce sont les «trésors humains vivants". Ils sont censés, en référence à l'article 2 alinéa 3 de la convention, être les dépositaires privilégiés d'un corps de connaissances, et les plus à même d'assurer la transmission d'un savoir. Selon les directives pour la création de systèmes nationaux de trésors humains vivants de l'Unesco, ce sont des «personnes ou groupes qui possèdent à un très haut niveau les connaissances et les savoir-faire nécessaires pour entretenir, créer ou produire des éléments spécifiques du patrimoine culturel immatériel. Ceux-ci sont choisis par leurs communautés et leurs États comme témoignages de leurs traditions culturelles vivantes et du génie créateur de communautés et de groupes présents sur leur territoire» (alinéa 2).

La Chine qui, sans surprise, élabore sa conception du $\mathrm{PCl}$ «avec des caractéristiques chinoises $\mathbf{1 2}^{\mathbf{2}}$, s'est inspirée de la notion de trésor humain vivant pour forger celle de «transmetteurs représentatifs » (daibiaoxing chuangchengren). L'article 29 de la récente «loi sur le patrimoine culturel immatériel de la République populaire de Chine» (2011) mentionne comme critères de sélection de ces derniers la maîtrise du patrimoine culturel visé, la représentativité et l'influence (notoriété) locales ${ }^{\mathbf{1 3}}$. Un transmetteur est en principe censé bénéficier d'un soutien financier de sa région et s'engager dans sa fonction de transmission, notamment auprès de disciples. En cas de décès, sa succession doit être assurée obligatoirement.

En RPC, avec un État qui, fidèle à une pratique séculaire, prend en charge la culture et, depuis les années 1950, opère sous l'égide d'une idéologie de parti, il semblerait approprié qu'un «transmetteur représentatif» soit un secrétaire du Parti. C'est le cas de Li Zisheng, «transmetteur » drung (dulong) de niveau provincial (shengji) pour sa communauté. II a été désigné transmetteur en 2007, plus spécifiquement pour la musique. II est l'un des 207 transmetteurs de rang provincial sélectionnés par le bureau de la culture de la province du Yunnan ${ }^{14}$. Voilà comment il est présenté dans l'ouvrage qui en fournit la liste: 
Li Zisheng, homme, de "nationalité » dulong [drung], est né en 1961. II est du village de Dizhengdang, du district autonome dulong et nu de Gongshan, préfecture autonome lisu du Nujiang. Il est un transmetteur pour la musique. Li Zisheng aime la musique et la danse populaire depuis son enfance, et dès ses 12 ans a appris à chanter les chansons populaires drung auprès de son père, et allait souvent écouter les plus vieux chanter à l'occasion de toutes sortes de réunions villageoises. À 20 ans, il pouvait chanter de nombreuses chansons dulong, et aussi danser. [...] II sait diriger la fête «Kaquewa» qui a lieu une fois l'an [...]. Li Zisheng maîtrise totalement l'art culturel traditionnel dulong, et a une notoriété et une influence certaines au sein de la communauté locale. (Yunnansheng Wenhuating [éd.] 2009: 163)

II est remarquable que Li Zisheng ait été choisi pour être le transmetteur de savoirs culturels et l'agent de leur perpétuation ${ }^{15}$. Secrétaire de cellule du Parti au niveau de son village, il a effectivement en tant que tel un rôle à jouer, comme nous l'avons vu, à l'interface des instances gouvernementales et de la société civile. Ce qui, à son niveau, se traduit essentiellement par les relations entre le gouvernement du canton et les habitans du village administratif (xingzhengcun) où il occupe son poste.

Mais, deux ans après sa nomination, la mort vint rapidement mettre un terme aux fonctions de "transmetteur" de Li Zisheng (une autre personne devrait être prochainement désignée pour occuper sa place). II joua néanmoins en son temps un rôle non négligeable. Nous avons vu comment il œuvra plus spécialement dans son village à la promotion du Kaquewa (kraltshang-wa), cet emblème de la culture drung. Cette fête n'était alors pas encore inscrite sur la liste nationale du patrimoine immatériel. Mais déjà, dans une démarche de type patrimonial, la «culture» en vint à désigner un bien, un ensemble de pratiques qui pouvaient être «mises en valeur», au sens plein du terme: valeur dans une économie d'échange de biens culturels, valeur comme symbole de ce qui peut être considéré comme digne de respect, propre à être sauvegardé.

Cette situation paradoxale du transmetteur défunt renvoie au caractère artificiel qu'une telle nomination peut revêtir. L'important est que celle-ci ait eu lieu, contribution supplémentaire à l'établissement de la relation métaculturelle aux objets de l'action patrimoniale. La capacité réelle dudit «transmetteur» à assurer la continuité des savoirs qu'il incarne semble seconde. Il est ainsi révélateur que le portrait de Li Zisheng figurant dans l'ouvrage de présentation des transmetteurs soit en fait celui d'une autre personne. Simple détail peut-être. En définitive, le transmetteur défunt n'est qu'un miroir de la fête disparue.

15. N'ayant pas d'informations directes sur le processus de sélection qui prit place au cours d'une période où je ne me suis pas rendu sur le terrain, je ne m'attarderai pas sur le bien-fondé du choix qui fait de ce secrétaire du Parti le dépositaire unique de telles connaissances. 


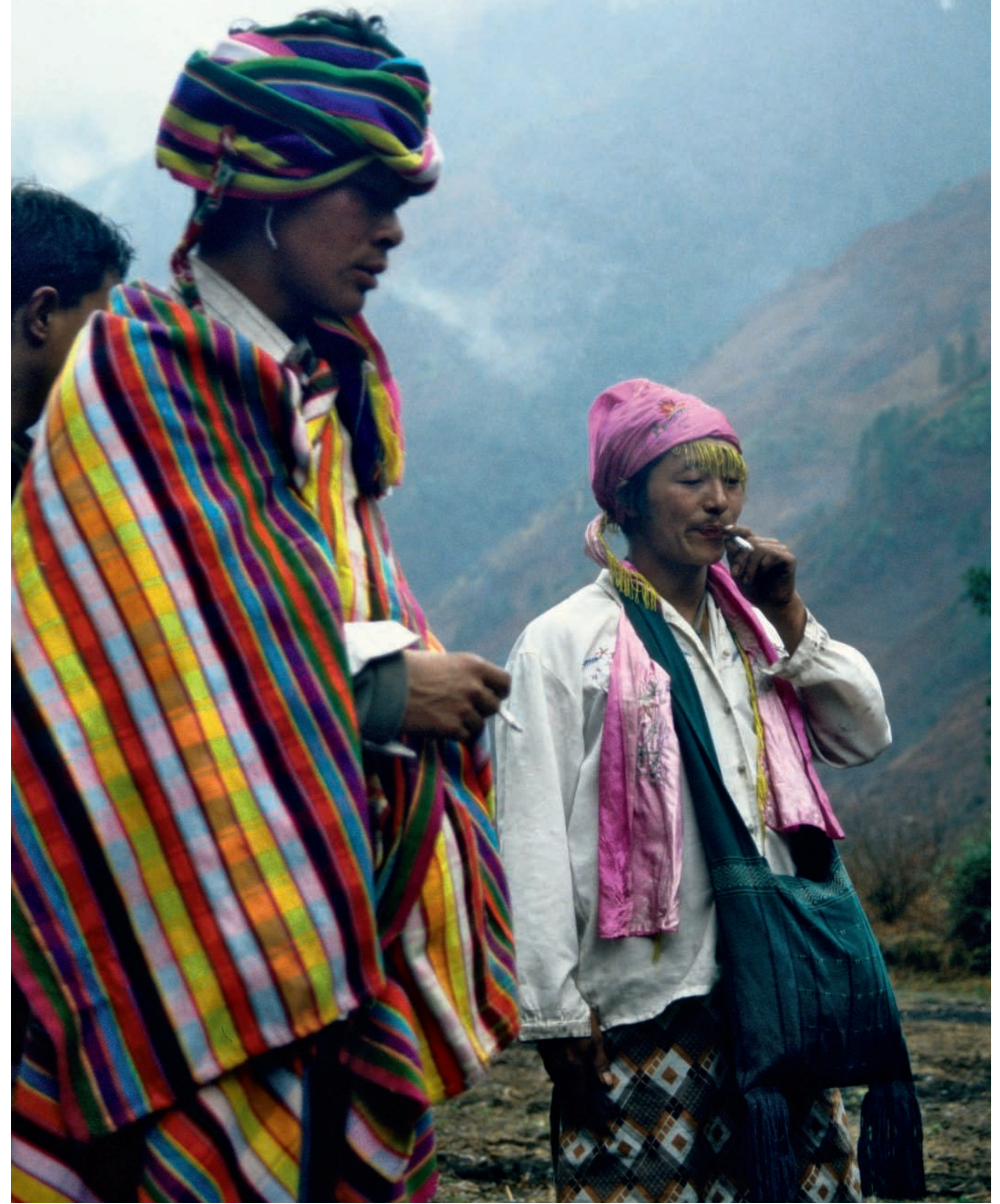




\section{Épilogue}

Au cours de l'hiver 2003, alors que les villageois de Dizhengdang se préparaient pour le dernier rituel de kraltshang-wa, je me rendis au village de Kongdang. Avec l'arrivée de la route carrossable en 2000, ce dernier était devenu le chef-lieu du canton de la vallée des Drung, abritant le gouvernement local et diverses administrations, et, depuis peu, de petits entrepreneurs et commerçants extérieurs à la vallée.

Les autorités locales se devaient d'organiser la tenue de la "fête drung", fixée au 10 janvier de chaque année. Quelques fonds publics y furent consacrés, notamment par le bureau de l'éducation. Une généreuse quantité d'alcool et la viande de deux cochons furent achetées, destinées à être distribuées gratuitement. Les villageois désirant se joindre à la fête vinrent à pied pour certains, à l'arrière d'un des camions d'un chantier proche pour d'autres. Chacun se servit d'alcool et de viande à faire griller sur le feu. Rassemblant finalement une cinquantaine de personnes, dont quelques responsables des administrations locales, la journée se déroula tout d'abord à manger, boire, discuter et jouer aux cartes. Puis un jeune instituteur démarra un petit générateur électrique pour alimenter une chaîne hi-fi qui gratifia l'assemblée d'une succession de musiques modernes sur lesquelles un certain nombre de participants se mirent à danser autour du feu. Avant la fin de l'après-midi, tout le monde rentra, par petits groupes, chez les uns ou les autres pour terminer la soirée à boire, danser et chanter en karaoké. La fête "Kaquewa" fut alors une occasion de réjouissance qui ne devait plus rien au kraltshang-wa. Elle n'en était pas même une pâle copie, une version folklorisée vidée de son contenu. Simple forme contemporaine et purement ludique d'un vague référent culturel, la fête n'est plus qu'emblème.

Si l'utilisation sans cesse croissante du terme de «patrimoine culturel immatériel " semble témoigner d'une «fièvre immatérielle", cet engouement pour une nouvelle catégorie s'inscrit en réalité dans la longue durée des politiques patrimoniales et des interventions de l'État dans le domaine de la culture. Ces politiques réinstituent la présence locale de l'État, avec parfois de nouveaux modes d'institutionnalisation de la culture: la notion vite adoptée en Chine de "patrimoine culturel immatériel » a peut-être plus simplement ouvert la porte à une nouvelle vague d'interventions. Inaugurant moins un renouvellement que la reproduction d'une économie des biens culturels, le $\mathrm{PCl}$ entretient avec la cause de la préservation une relation ambiguë. Du Kaquewa, il ne préserve que la perte.

Le cas de la fête drung est un exemple du formalisme des travaux d'identification et de la réalisation d'inventaires liés à la mise en œuvre de la convention en Chine. Certes, la notion de $\mathrm{PCl}$ a permis, dans une certaine mesure, de contourner les catégories moralisatrices et les notions péjoratives de "superstition", ou encore de "féodalité" et de "primitivisme". Elle a également donné à divers acteurs au niveau national la possibilité d'engager des discussions autour de l'histoire, de la tradition et de tout ce qui entrait communément dans la catégorie officielle de la culture populaire (minjian wenhua). Mais, comme je l'ai souligné, ce régime culturel était déjà bien en place dans les années qui ont précédé son usage.

ci-contre

fig. 6

Les hommes déguisés et travestis, 2003. Photo Stéphane Gros. 


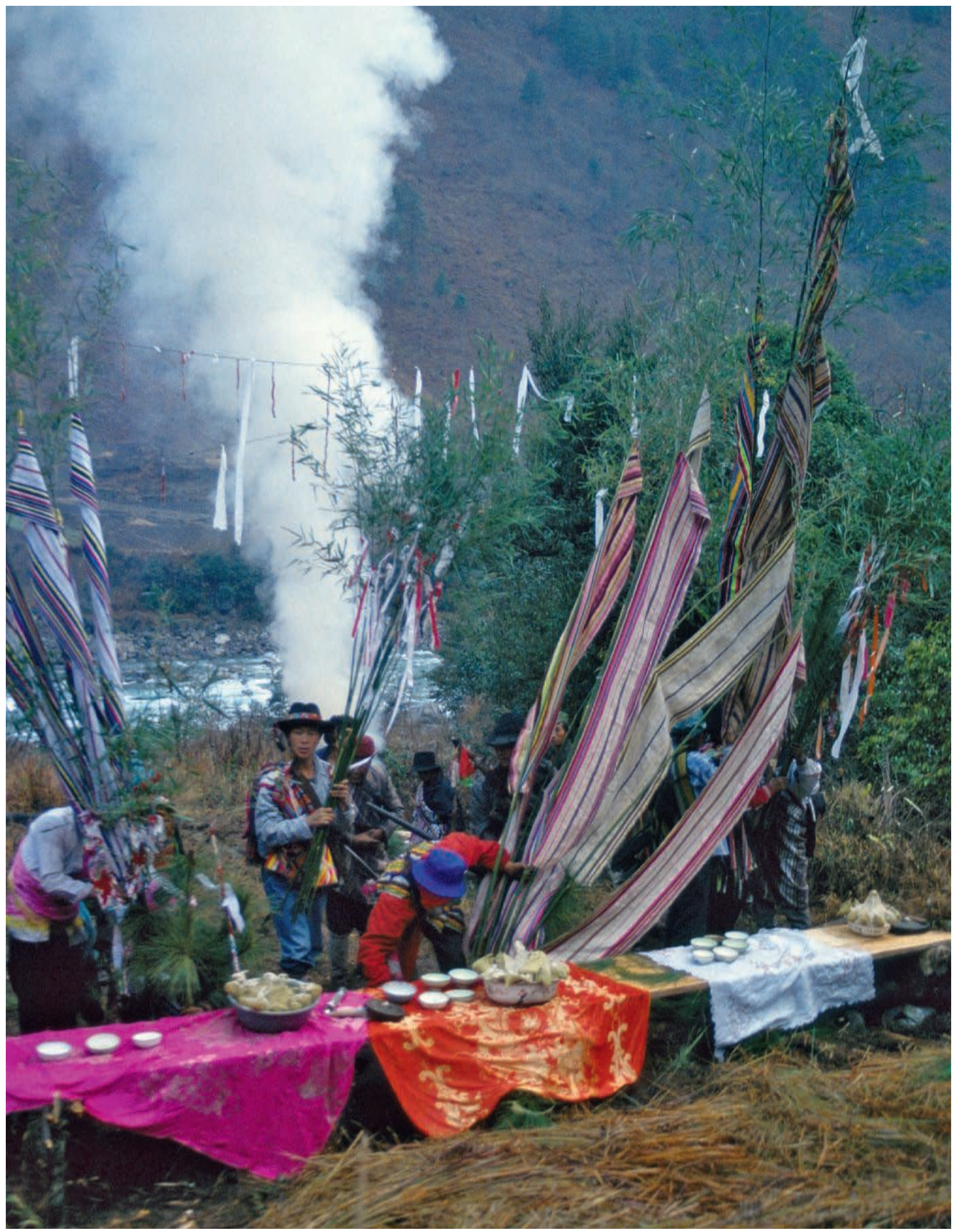


Cet élément patrimonial qu'est le Kaquewa représente de manière paradigmatique - quoique exceptionnelle - l'effet d'éternité produit par l'inventaire, rituel d'État. À la présumée continuité de la transmission s'impose la gestion quasi muséographique du vivant. La figure du «trésor humain vivant", ou autre "transmetteur", n'est pas sans évoquer dans le contexte chinois un parallèle avec celle du "fossile vivant "; notion souvent utilisée en Chine, dérivée de l'évolutionnisme social et appliquée aux coutumes ou aux peuples en tant que «survivances" d'états antérieurs, et justement trésors de culture parce qu'ils seraient la relique d'un passé révolu. Ces éléments deviennent en somme "les miroirs ou les écrans d'une perte durable ${ }^{16}$ " (Ciarcia 2006: 6), emblèmes patrimoniaux de non-lieu de la transmission culturelle.

Car, plus fondamentalement, comment comprendre le désir des villageois d'abandonner leurs pratiques rituelles? En particulier celle qui, une fois l'an, visait autrefois à assurer le bien-être de la communauté et donner les moyens de la reproduction sociale? En effet, quand bien même certains décidèrent de refaire le Nouvel An, ce ne fut que pour une durée limitée. Les villageois, dans leur ensemble, s'entendent pour ne plus investir le rituel annuel du rôle moteur qui fut le sien, n'y voyant plus de réel enjeu et en acceptant déjà l'inéluctable perte pour placer désormais en d'autres lieux leurs espoirs de reconstruction sociale.
16. C'est Gaetano Ciarcia qui souligne.

\section{ci-contre}

fig. 7

Préparation des offrandes devant l'autel, 2003.

Photo Stéphane Gros.

\section{remerciements}

Cet article a grandement profité des remarques formulées par de nombreux collègues sur des versions antérieures à l'occasion de présentations orales au Laboratoire d'Anthropologie et d'Histoire de l'Institution de la Culture (juin 2009), à l'atelier The Entangled Ethnographer, Centre for Research in the Arts, Social Sciences and Humanities, Université de Cambridge (juin 2010) et au Centre d'études himalayennes (mai 2012). Je les remercie vivement, ainsi que les coordinatrices de ce dossier. 
Bibliographie

\section{Babadzan, Alain}

2001 «Les usages sociaux du patrimoine", Ethnologies comparées 2 [en ligne] ; http://alor. univ-montp3.fr/cerce/r2/a.b.htm.

\section{Barbe, Noël}

2007 "Le déploreur de l'utilité, l'expert fraternel et l'inventeur de science détaché. Production de savoir et action culturelle. Prolégomènes... » Ethnographiques.org 12 [en ligne] ; http://www. ethnographiques.org/2007/Barbe.

\section{Barmé, Geremie}

2006a "Editorial», China Heritage Quaterly 7 [en ligne] ; http://www. chinaheritagequarterly.org/editorial. php?issue $=007$.

2006b «A tale of two lists: An examination of the new lists of intangible cultural properties » China Heritage Quaterly 7

[en ligne] ; http://www. chinaheritagequarterly.org/features. php? searchterm $=007$ twolists. inc\&issue=007.

\section{Bortolotto, Chiara}

2011 « Introduction. Le trouble du patrimoine culturel immatériel », in Chiara Bortolotto (dir.), avec la collaboration d'Annick Arnaud et Sylvie Grenet, Le Patrimoine culturel immatériel. Enjeux d'une nouvelle catégorie. Paris, Éditions de la Maison des sciences de l'homme (« Ethnologie de la France » 26): $21-43$

\section{Briggs, Charles}

1996 "The politics of discursive authority in research on the "invention of tradition" ", Cultural Anthropology 11(4): 435-469.

L’injonction à la fête. Par Stéphane Gros

\section{Brosius, Christiane et Polit, Karin (éd.)}

2011 Ritual, Heritage and Identity. The Politics of Culture and Performance in a Globalised World. Londres, Routledge.

\section{Ciarcia, Gaetano}

2006 La Perte durable.

Étude sur la notion de "patrimoine immatérie/». Paris, Lahic/Mission à l'ethnologie (“Carnet du Lahic » 1).

\section{Conseil des affaires} d'État

2006 « Guowuyuan guanyu gongbu diyipi guojiaji feiwuzhi wenhua yichan minglu de tongzhi », [en ligne]; www.ihchina.cn/inc/ detail.jsp?info_id=203.

\section{Fresnais, Jocelyne}

2001 La Protection du patrimoine en République populaire de Chine, 1949-1999. Paris, Éditions du CTHS.

\section{Geertz, Clifford}

1960 «The Javanese Kijaji: The changing role of a cultural broker », Comparative Studies in Society and History 2(2): 228-249.

\section{Gros, Stéphane}

2010 "A Heuristic blunder. Notes on an ethnographic situation in Northwest Yunnan (China)", Asia Pacific Viewpoint 51(2): 148-163.

2012a La Part manquante. Échanges et pouvoirs chez les Drung du Yunnan (Chine). Nanterre, Société d'ethnologie.

2012b « Cultes de fertilité chez les Drung du Yunnan ", Moussons 19: 111-136.

\section{Naepels, Michel}

1998 «Une étrange étrangeté. Remarques sur la situation ethnographique », L'Homme 148 185-200.

\section{Nas, Peter J.M.}

2002 «Materpieces of oral and intangible culture. Reflections on the Unesco World Heritage list », Current Anthropology 43(1): 139-148.

\section{Pan, Shouyong}

2008 «Museums and the protection of cultural intangible heritage ", Museum International 60(1-2).

\section{Tornatore, Jean-Louis}

2007 «Qu'est-ce qu'un ethnologue politisé? Expertise et engagement en socio-anthropologie de l'activité patrimoniale"

Ethnographiques.org 12 [en ligne] www.ethnographiques.org/2007/ Tornatore.

\section{Trebinjac, Sabine}

1990 «Que cent chants rivalisent, qu'une musique éclose, étude sur le traditionalisme d'État en Chine", Archives européennes de sociologie 31(1): 60-99.

\section{Yunnansheng Wenhuating (éd.)}

2009 Yunnansheng feiwuzhi wenhua yichan chuanchengren minglu. Kunming, Yunnan Daxue Chubanshe.

\section{Zhang, Liang}

2003 La Naissance du concept de patrimoine en Chine, $x I X^{e}-x x^{e}$ siècles.

Paris, Éditions Recherches/ Ipraus. 
